\title{
THE MEASUREMENT OF REFLECTION AND TRANSMISSION FACTORS
}

\author{
By M. Luckiesh
}

Little has been done toward standardizing the determination of reflection and transmission factors, but owing to the fact that there are a number of conditions which influence such measurements this should be done. These values are ordinarily presented with such nonchalance that one is led to conclude there is a general belief that they are fixed and invariable quantities possessed by the surfaces or media for which they have been determined. In two papers ${ }^{1}$ dealing respectively with the two quantities it has been the aim to show the conditions which influence the results and to determine the methods which should be standardized for such measurements. It is the aim of this paper to review the problems briefly and to propose a standardization of methods.

\section{REFLECTION FACTOR}

Surfaces generally reflect light both specularly and diffusely; that is, they are usually far from perfect mirrors or perfect diffusers. Assuming the general surface, the following are the chief factors which influence the value determined by measurement:

I. Character of the reflecting surface.

It is not unusual to determine the reflection factor by comparing the brightness of the surface with that of a surface of known reflection factor under the same intensity of illumination. If the surface is highly glazed it is obvious that the brightness will be made up of that due to diffuse reflection and that due to a more or less perfect image of the light-source or of other luminous objects mirrored in the glazed surface. The "specular brightness" of the surface varies with the brightness of the light-source or other mirrored object, whereas the "diffuse brightness" depends upon the intensity of illumination. Hence specular reflection may introduce a spurious quantity into the determination of reflection factor. The values of reflection factor for a given surface as determined in this manner may differ considerably, due to variation in the brightness of the source of illumination.

${ }^{1}$ M. Luckiesh, Measurement of Reflection-Factor, Elec. World, May 19, 1917. M. Luckiesh and L. L. Mellor, Measurement of Transmission-Factor, Jour. Frank. Inst. 186, 1918, p. 529. 
2. Location of the source of illumination.

If the light-source is so located that light is specularly reflected into the photometer which is used for making a comparison of the brightnesses of the specimen with that of a standard surface, the difficulty described in the preceding paragraph will be encountered. In the case of glossy surfaces, slight changes in the position of the photometer, of the light-source, or of the surface may alter the brightness determination considerably, and it is obvious that different values of reflection factor will be obtained. Each value is correct for the specific conditions, but a statement of conditions is necessary. Therefore it is desirable to have conditions which are easily described and reproduced.

3. Solid-angular extent of the light-source.

As already stated the specularly reflected image will vary, for a given surface, with the brightness of the light-source. The latter can vary widely and still the illumination, and also the component of brightness due to diffusely reflected light, may remain constant.

\section{Spectral character of the illuminant.}

It is not uncommon to have the reflection-factors of colored surfaces, such as wall-papers and paints, presented without specifying the illuminant. The reflection factor of a colored surface may vary as much as 50\% for the illuminants ordinarily encountered. The illuminant should always be specified sufficiently so that the spectral distribution of energy is indicated.

5. The method of photometry.

In the case of considerable differences in color, the two chief methods of photometry, namely the direct-comparison and the flicker methods, will yield different results. This is one of the most annoying aspects, but in the absence of standardization, the method of photometry should be specified, and other conditions such as the size of the photometric field and the approximate absolute brightness should be recorded. Perhaps the flicker photometer should be generally standardized for the measurement of reflection and transmission factors owing to the better reproducibility of measurements by this method. However, there are still many unsettled questions pertaining to color photometry. 

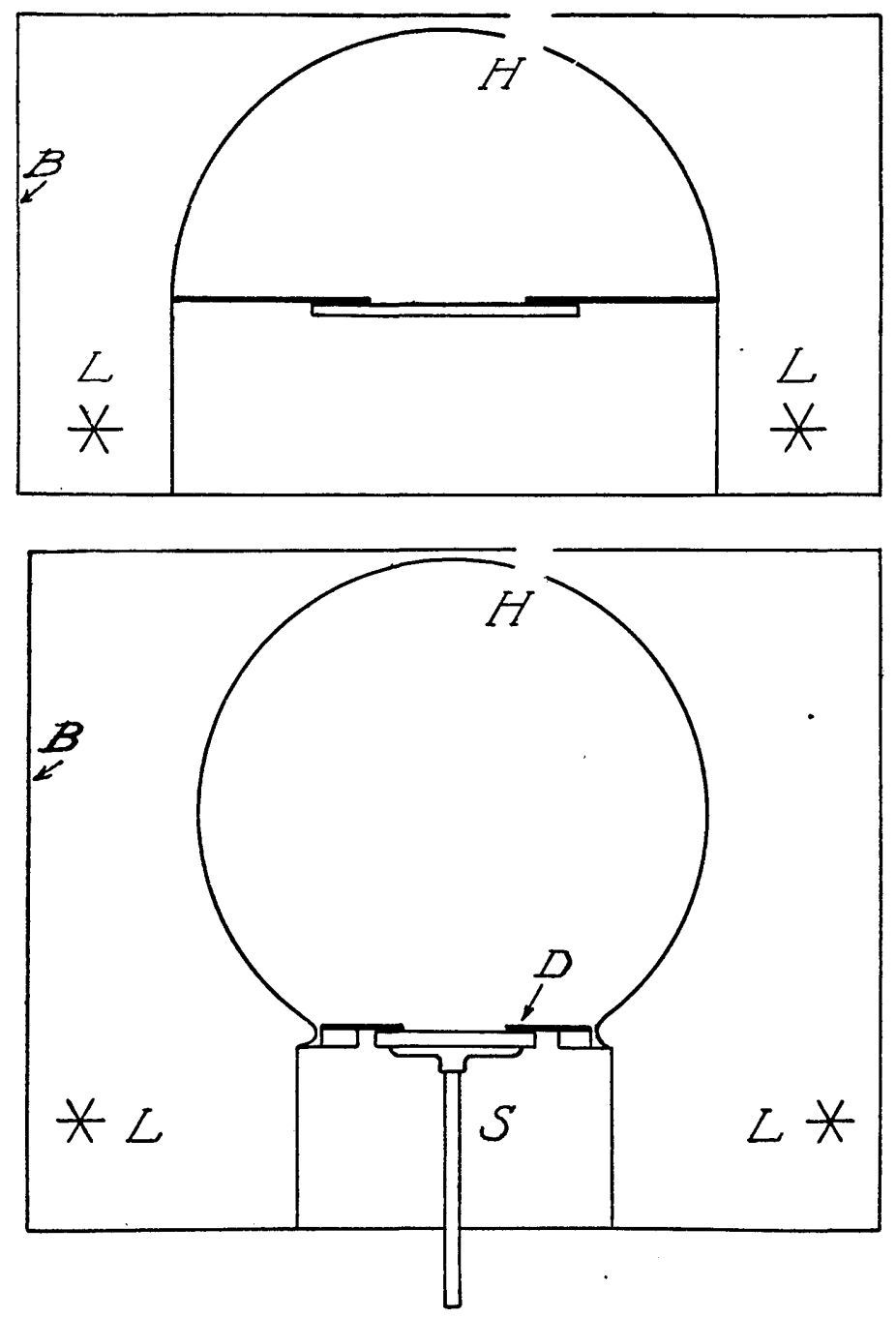

Fig. 1-Apparatus for determining reflection-factors of specimens diffusely illuminated.

In general these influences can not be avoided, but the method selected for standardization should be one which is equitable and easily described, and one which represents fairly well the conditions under which the reflection factor of the surface is of interest in its practical use. Generally we are concerned with the reflection factor of the surface as a whole, that is, a sort of weighted mean of the reflection factors for all directions. In case a more intimate analysis of the reflecting characteristic of the surface is desired, a reflection-factor curve for all angles may be determined. 
It appears that perfectly diffused illumination best meets the requirements for determining the reflection factor of a surface as a whole. In other words the light-source should be of uniform brightness and should subtend an entire hemisphere. A paint or wall-paper in actual use receives light from many directions and seldom is its reflection factor for a certain direction or angle of interest. Except in the case of glossy surfaces of low reflection factor the amount of light specularly reflected is much smaller than that which is diffusely reflected. By using perfectly diffuse illumination the specular brightness is not permitted to contribute disproportionately to the total brightness. If a narrow beam of light is used and the photometer is placed in a position to avoid specular reflection the specularly reflected component is entirely eliminated, which obviously is unjust unless only the diffuse reflection factor is desired. These questions arise, for example, in comparing the reflection factors of paints and a difference of a few per cent may do an injustice to some samples.

The Nutting reflectometer in which is employed the practical equivalent of two paralled surfaces of infinite extent and of uniform brightness, meets the foregoing suggestion; that is, the illumination of the specimen whose reflection factor is desired is practically perfectly diffuse, the lightsource subtending in effect an entire hemisphere. The Martens polarization photometer, which Nutting originally used with his ingenious device, could be replaced by some other compact optical instrument. Ordinary photometers are more generally available, and it is easy to provide a simple apparatus similar to one of those illustrated in Fig. I for the purpose of obtaining diffuse illumination. A dense opal glass globe or hemispherical shell is provided with a hole $(\mathrm{H})$ and placed as shown in a box (B) painted white. A number of lamps (L) are symmetrically located on the bottom of the box. The samples to be tested are pressed upward against a thin metal diaphragm (D) with a suitable aperture in it. The hole $(\mathrm{H})$ should be offset somewhat in order to avoid reflecting an image of it or of the nose of the photometer into the photometric field. These two devices have been used successfully and the results obtained check well with those determined with the Nutting reflectometer. In using the devices illustrated in Fig. I, it is obvious that a surface of known reflection factor should be available so that the reflection factors of the specimens may be obtained by a direct comparison of brightnesses. The reflection factor of the standard may be determined by means of the Nutting reflectometer which yields absolute 
values, or if it is a perfectly diffusely reflecting surface the simple relation between illumination and brightness can be utilized. White blotting paper or sandblasted opal glass are satisfactory working standards.

The integrating sphere can be utilized for determining the reflection factors of reflecting surfaces by placing the latter inside the sphere and permitting a beam of light to enter and illuminate the reflecting surface. A suitable arrangement of the various details presents no difficulties.

\section{TRANSMISSION FACTOR}

Similar influences bear upon the results of the determination of transmission factors as of reflection factors, but there is another point to be considered which does not apply to reflecting surfaces, namely,

6. The transmission factor depends, in the case of one side of the specimen being rough, upon the direction in which the light passes through it.

For example, the transmission factor is generally smaller when the smooth surface faces the light-source than when the rough side faces the light-source, other conditions remaining equal. This difference is greatest for a narrow beam of light and diminishes as the illumination becomes more diffuse, usually disappearing when the illumination has become perfectly diffuse.

Some of these differences are shown in the accompanying table for flat crystal glass specimens smooth on one side. Specimen 5 was a sheet of clear glass with one side sand-blasted; specimen 7 was a sheet of clear glass etched with acid on one side; specimen 8 was a common "pebbled" glass, smooth on one side; the remaining specimens contained ribs on one side the other side being smooth. For further details, reference should be made to the original paper. It is seen that the transmission factor is different for a narrow beam of light at perpendicular incidence than for diffuse illumination. The ratio of the transmission factor for a narrow beam to that for diffuse illumination is given in the last column for each case. In general it appears best to employ perfectly diffuse illumination for the determination of transmission factors and this can be done by an arrangement of apparatus similar to that illustrated in Fig. 2. $\mathrm{H}$ is a hemispherical shell of opal glass; $\mathrm{L}$ are light-sources, located symmetrically with respect to $\mathrm{H}$; $\mathrm{O}$ is an opening into a small intergrating sphere $\mathrm{I} 5$ inches in diameter; $\mathrm{T}$ is a target, painted white on both sides; $B$ is a box, painted white inside; and the photometer is placed underneath. The specimen to be tested is placed over $\mathrm{O}$, just under 

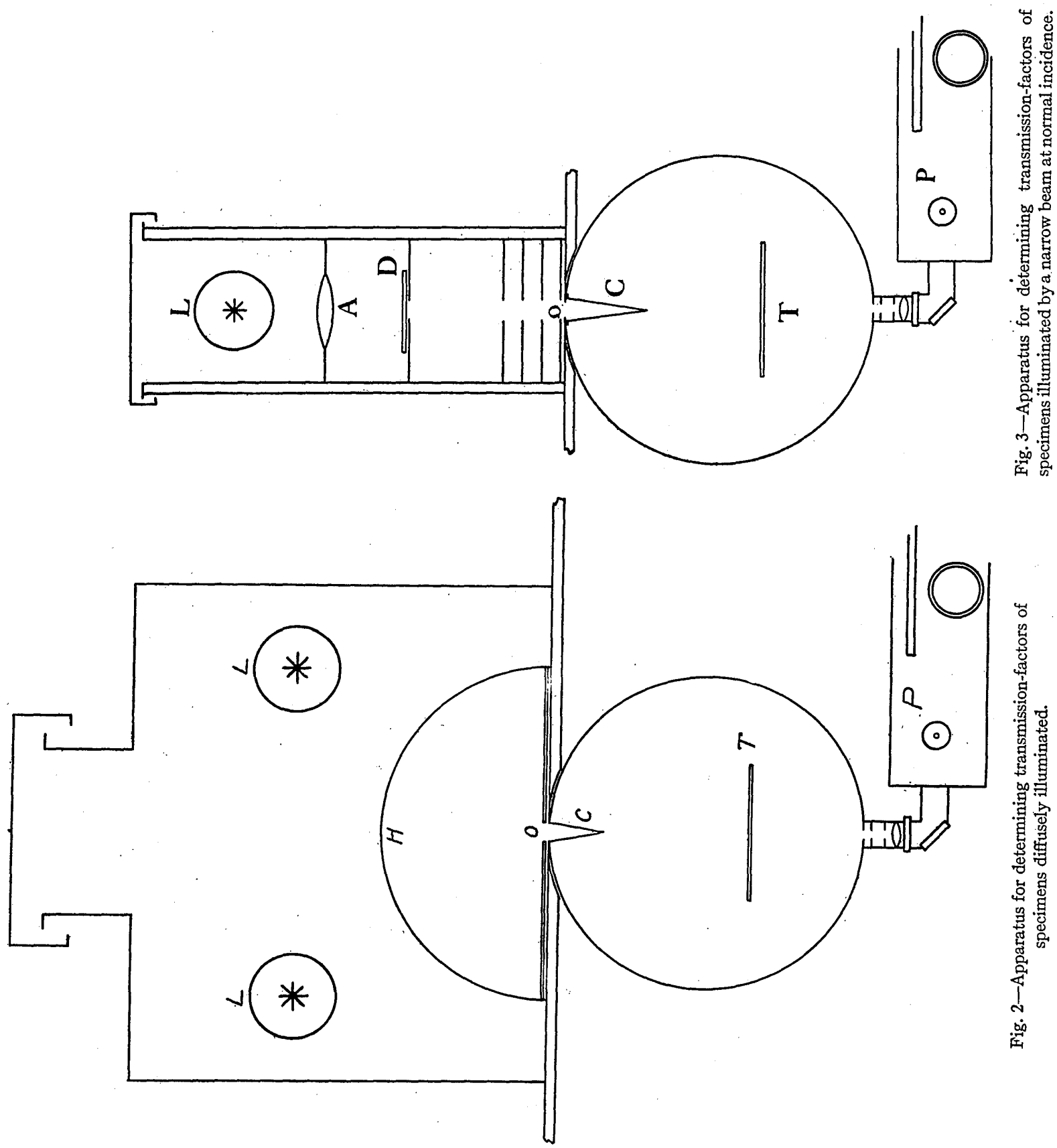

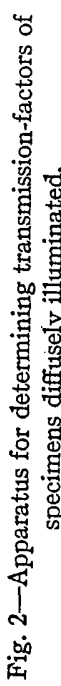


a thin diaphragm with a hole in it. Provision should be made for having the two sides of the specimen in contact with the diaphragm and $\mathrm{O}$ respectively. A disadvantage of this scheme is the great amount of light required.

In case a narrow pencil of light is desired, the apparatus shown diagrammatically in Fig. 3 is satisfactory. $\mathrm{L}$ is a single powerful light source; $\mathrm{A}$ a lens; and $\mathrm{D}$ a sheet of clear glass, etched on one side. C is a slender, hollow cone of clear glass, acid etched on its exterior. An extensive investigation indicated the necessity. of such a device for the purpose of partially diffusing the light entering the sphere. Satisfactory proof was obtained that this cone did not introduce any error of measurable magnitude. If the transmission, characteristic of a specimen is desired, the sphere and cone may be removed, and the relative intensity of transmitted light may be determined for the angles desired. Obviously other modifications of the integrating sphere can be utilized.

Transmission Factors for Crystal Glasses Under Various CONDITIONS

\begin{tabular}{|c|c|c|c|c|}
\hline \multirow{2}{*}{ Specimen of glass } & \multirow{2}{*}{$\begin{array}{l}\text { Side toward } \\
\text { light source }\end{array}$} & \multicolumn{2}{|c|}{ Transmission Factor } & \multirow{2}{*}{$\begin{array}{l}\text { Ratio } \\
\frac{\text { diffuse }}{\text { direct }}\end{array}$} \\
\hline & & $\begin{array}{c}\text { Narrow } \\
\text { beam }\end{array}$ & $\begin{array}{l}\text { Perfectly } \\
\text { diffuse }\end{array}$ & \\
\hline 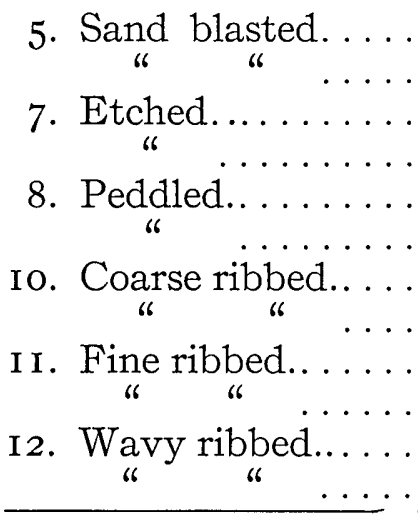 & $\begin{array}{l}\text { Rough } \\
\text { Smooth } \\
\text { Rough } \\
\text { Smooth } \\
\text { Rough } \\
\text { Smooth } \\
\text { Ribbed } \\
\text { Smooth } \\
\text { Ribbed } \\
\text { Smooth } \\
\text { Ribbed } \\
\text { Smooth }\end{array}$ & $\begin{array}{r}0.783 \\
.739 \\
.794 \\
.758 \\
.846 \\
.790 \\
.766 \\
.5 \mathrm{I} 5 \\
.858 \\
.790 \\
.884 \\
.860 \\
\end{array}$ & $\begin{array}{r}0.702 \\
.695 \\
.709 \\
.704 \\
.746 \\
.746 \\
.617 \\
.616 \\
.793 \\
.791 \\
.822 \\
.821\end{array}$ & $\begin{array}{r}0.897 \\
.940 \\
.893 \\
.929 \\
.882 \\
.944 \\
.805 \\
\text { I. I96 } \\
.924 \\
\text { I. } .001 \\
.929 \\
.955\end{array}$ \\
\hline
\end{tabular}

SUMMARY

The aims of this paper are to indicate the conditions which influence the results of determinations of reflection and transmission factors; to indicate the desirability of standard methods; and to recommend such standard methods. In general it is recommended that reflection and transmission factors be determined for diffuse illumination; that is, that a light-source of uniform brightness subtending a hemispherical angle should be used. 